\title{
Article \\ Serum miRNAs Support the Indication for MRI-Ultrasound Fusion-Guided Biopsy of the Prostate in Patients with Low-PI-RADS Lesions
}

\author{
Bastian Keck 1,+, Angelika Borkowetz ${ }^{2,+}$, Julia Poellmann 1,3, Thilo Jansen 1,3, Moritz Fischer 1,3 ${ }^{\text {(D), }}$ \\ Susanne Fuessel ${ }^{2,4}$, Andreas Kahlmeyer 1,3, Manfred Wirth ${ }^{2}$, Johannes Huber ${ }^{2} \mathbb{D}$, Alexander Cavallaro ${ }^{3,5}$, \\ Matthias Hammon ${ }^{(D)}$, Ivan Platzek ${ }^{6}$, Arndt Hartmann ${ }^{3,7}$, Gustavo Baretton ${ }^{8}$, Frank Kunath 1,3, Danijel Sikic 1,3, \\ Helge Taubert ${ }^{1,3, * \mathbb{D}}$, Bernd Wullich ${ }^{1,3}$, Kati Erdmann ${ }^{2,4,9, \ddagger(\mathbb{D})}$ and Sven Wach $1,3,4, \ddagger$
}

1 Department of Urology and Pediatric Urology, University Hospital Erlangen, Friedrich-Alexander Universität Erlangen, Krankenhausstrasse 12, 91054 Erlangen, Germany; bastian.keck@web.de (B.K.); julia.poellmann@uk-erlangen.de (J.P.); thilo.jansen@googlemail.com (T.J.); moritzfischer2001@yahoo.de (M.F.); andreas@dr-kahlmeyer.de (A.K.); frank.kunath@uk-erlangen.de (F.K.); danijel.sikic@uk-erlangen.de (D.S.); Bernd.Wullich@uk-erlangen.de (B.W.); sven.wach@uk-erlangen.de (S.W.)

2 Department of Urology, Medical Faculty Carl Gustav Carus, Technische Universität Dresden, Fetscherstrasse 74, 01307 Dresden, Germany; Angelika.Borkowetz@uniklinikum-dresden.de (A.B.); Susanne.Fuessel@uniklinikum-dresden.de (S.F.); Manfred.Wirth@uniklinikum-dresden.de (M.W.); Johannes.Huber@uniklinikum-dresden.de (J.H.); Kati.Erdmann@uniklinikum-dresden.de (K.E.)

Citation: Keck, B.; Borkowetz, A.; Poellmann, J.; Jansen, T.; Fischer, M.; Fuessel, S.; Kahlmeyer, A.; Wirth, M.; Huber, J.; Cavallaro, A.; et al. Serum miRNAs Support the Indication for MRI-Ultrasound Fusion-Guided Biopsy of the Prostate in Patients with Low-PI-RADS Lesions. Cells 2021, 10, 1315. https://doi.org/10.3390/ cells10061315

Academic Editors: Hiroshi Miyamoto and Qiming Jane Wang

Received: 12 April 2021

Accepted: 21 May 2021

Published: 25 May 2021

Publisher's Note: MDPI stays neutral with regard to jurisdictional claims in published maps and institutional affiliations.

Copyright: () 2021 by the authors. Licensee MDPI, Basel, Switzerland. This article is an open access article distributed under the terms and conditions of the Creative Commons Attribution (CC BY) license (https:// creativecommons.org/licenses/by/ $4.0 /)$.
3 Comprehensive Cancer Center Erlangen-EMN (CCC ER-EMN), Östliche Stadtmauerstrasse 30 91054 Erlangen, Germany; alexander.cavallaro@uk-erlangen.de (A.C.); arndt.hartmann@uk-erlangen.de (A.H.)

4 Member of the Association of Scientists in Urological Research (UroFors) of the German Society of Urology, Martin-Buber-Straße 10, 14163 Berlin, Germany

5 Department of Radiology, University Hospital Erlangen, Friedrich-Alexander Universität Erlangen, Maximiliansplatz 3, 91054 Erlangen, Germany; matthias.hammon@gmail.com

6 Department of Radiology and Interventional Radiology, Medical Faculty Carl Gustav Carus, Technische Universität Dresden, Fetscherstrasse 74, 01307 Dresden, Germany; Ivan.Platzek@uniklinikum-dresden.de

7 Institute of Pathology, University Hospital Erlangen, Friedrich-Alexander Universität Erlangen, Krankenhausstrasse 8-10, 91054 Erlangen, Germany

8 Institute of Pathology, Medical Faculty Carl Gustav Carus, Technische Universität Dresden, Fetscherstrasse 74,01307 Dresden, Germany; Gustavo.Baretton@uniklinikum-dresden.de

9 National Center for Tumor Diseases (NCT), Fetscherstrasse 74, 01307 Dresden, Germany

* Correspondence: helge.taubert@uk-erlangen.de; Tel.: +49-9131-8542658; Fax: +49-9131-8523374

+ First authors contributed equally.

$\ddagger$ Last authors contributed equally.

Abstract: Multiparametric MRI (mpMRI) and targeted biopsy of the prostate enhance the tumor detection rate. However, the prediction of clinically significant prostate cancer (PCa) is still limited. Our study tested the additional value of serum levels of selected miRNAs in combination with clinical and mpMRI information for PCa prediction and classification. A total of 289 patients underwent targeted mpMRI-ultrasound fusion-guided prostate biopsy complemented by systematic biopsy. Serum miRNA levels of miRNAs (miR-141, miR-375, miR-21-5p, miR-320b, miR-210-3p, let-7c, and miR-486) were determined by quantitative PCR. Detection of any PCa and of significant PCa were the outcome variables. The patient age, pre-biopsy PSA level, previous biopsy procedure, PI-RADS score, and serum miRNA levels were covariates for regularized binary logistic regression models. The addition of miRNA expression of miR-486 and let-7c to the baseline model, containing only clinical parameters, increased the predictive accuracy. Particularly in patients with PI-RADS $\leq 3$, we determined a sensitivity for detecting significant PCa (Gleason score $\geq 7$ a corresponding to Grade group $\geq 2$ ) of $95.2 \%$, and an NPV for absence of significant PCa of $97.1 \%$. This accuracy could be useful to support patient counseling in selected cases.

Keywords: diagnosis; microRNA; miRNA; mpMRI; PI-RADS; prostate biopsy; prostate cancer 


\section{Introduction}

Multiparametric magnetic resonance imaging (mpMRI) and targeted biopsy of tumorsuspicious lesions have become established diagnostic tools for the detection of prostate cancer (PCa) [1], since ultrasound-guided biopsy alone misses approximately $25-30 \%$ of PCa cases [2,3]. MpMRI has demonstrated a higher sensitivity and specificity for the detection of PCa [4] and is widely accepted among healthcare practitioners [5].

An increasing amount of published data has shown that targeted MRI-ultrasound fusion-guided biopsies have the potential to reduce the diagnosis of insignificant PCa and to enhance the detection rate of clinically significant PCa while reducing the number of biopsies, as the Prostate Imaging Reporting and Data System (PI-RADS) has a good diagnostic accuracy and correlation with PCa aggressiveness [6,7]. Clinical parameters, such as the pre-biopsy serum PSA level and mpMRI of the prostate, represent the basis of the clinical information used for decision making and patient counseling.

Nevertheless, in clinical practice, the diagnosis of PCa is accompanied by various uncertainties. Therefore, additional factors, not only enhancing the prediction of tumor presence but also tumor classification, are an important clinical need. We have previously shown that miRNAs are consistently deregulated in PCa and, by regulating their cognate protein targets, contribute to prostatic carcinogenesis [8,9]. Moreover, we and others could demonstrate that miRNAs derived from whole blood [10] or blood serum [11-14] can serve as biomarkers with the potential to differentiate PCa patients from patients with benign prostatic hyperplasia. However, it is not fully understood whether different miRNAs originate from tumor cells or if they are rather a response of the host organism to the presence of PCa [15]. Therefore, we set up a prospective MRI-guided biopsy study supplemented with serum-miRNA-analysis to evaluate the additional predictive value of the miRNA analysis to the clinical factors. Hereby, the addition of serum-based miRNA analysis to the clinical routine factors was able to enhance the predictive values of the mathematical models for predicting significant PCa.

\section{Materials and Methods}

\subsection{Study Population}

For the discovery cohort, a total of 80 consecutive patients with suspicion of PCa were recruited between January 2015 and July 2016. Patients were referred to the University Hospital Erlangen for targeted MRI-ultrasound fusion-guided prostate biopsy, which was complemented by systematic biopsy. Prostate mpMRIs were performed on 3T devices (Siemens Medical Solutions, Erlangen, Germany). All image sets were examined by an experienced radiologist and were scored according to PI-RADS V2.0. A combined targeted and systematic 12-core transrectal MRI-ultrasound fusion-guided biopsy was performed using the General Electric LOGIQ E9 (GE Healthcare, Solingen, Germany) system. Suspicious regions (PI-RADS scores 3, 4, 5) were biopsied specifically and non-suspicious regions (PI-RADS scores 1,2) systematically. Written informed consent was obtained before biopsy and the study was performed according to the Declaration of Helsinki. Ethical approval was provided by the ethics institutional review board of the University Hospital Erlangen (No. 3755, dated February 2008).

For the validation cohort, a total of 209 consecutive patients with suspicion of PCa were recruited between January and December 2015. Patients were referred to the University Hospital Dresden for targeted MRI-ultrasound fusion-guided prostate biopsy. Prostate mpMRIs were performed on 3T devices (Siemens Medical Solutions, Erlangen, Germany). All image sets were examined by two experienced radiologists and scored according to PI-RADS V.2.0. At this study site, the BioJet-System (d\&k Technologies, Barum, Germany) was used for MRI-ultrasound fusion-guided biopsy as described previously [16]. Briefly, fusion-guided prostate biopsy was performed in a transperineal approach, taking at least two cores per lesion. Lesions classified as PI-RADS $\geq 2$ were biopsied in a targeted fashion. Subsequently, every patient underwent a transrectal 12-core systematic biopsy. Written informed consent was obtained before biopsy. Ethical approval was provided by the ethics 
institutional review board of the Technische Universität Dresden (No. EK194092004, dated July 2009).

According to the EAU guidelines, both diagnostic procedures are regarded as equal in their diagnostic performance [17]. The clinical end points examined were histologically confirmed PCa and clinically significant $\mathrm{PCa}$ (csPCa), defined as Gleason score $\geq 7 \mathrm{a}$ (corresponding to Grade group $\geq 2$ ). We followed a holistic approach with histologic diagnosis as the final outcome. In clinical practice, it is not relevant if significant PCa was found in a targeted or non-targeted biopsy core.

\subsection{Blood Sampling and RNA Isolation}

Before biopsy, venous blood was drawn into coagulation tubes (Sarstedt, Nümbrecht, Germany) and further processed within two hours. Serum was prepared from the coagulated blood by centrifugation ( $2000 \mathrm{~g}$ for $10 \mathrm{~min}$ ) and samples were stored in aliquots at $-80^{\circ} \mathrm{C}$. Serum miRNAs were prepared from $200 \mu \mathrm{L}$ of serum with the miRCURY RNA Isolation Kit for biofluids (Exiqon, Vedbaek, Denmark) in the discovery cohort and the miRNA plasma kit (Promega, Madison, WI, USA) using an automated Maxwell RSC device (Promega) in the validation cohort, according to the respective manufacturer's recommendations.

\subsection{Quantitative PCR}

Synthesis of cDNA and subsequent quantification of miRNAs was conducted using TaqMan reverse transcription reagents and miRNA-specific TaqMan microRNA Expression Assays for miR-21-5p (ID: 000397), miR-141-3p (ID: 000463), miR-210-3p (ID: 000512), miR320b (ID: 002844), miR-375-3p (ID: 000564), miR-486-5p (ID: 001278) let-7c-5p (ID: 000379), and miR-16-5p as reference (ID: 000391) (Thermo Scientific, Darmstadt, Germany) according to the manufacturer's recommendations. Briefly, a constant volume of $4 \mu \mathrm{l}$ of isolated serum RNA was reverse transcribed in a total volume of $15 \mu$ l using a customized pool of the respective miRNA-specific stem-loop primers and the TaqMan MicroRNA Reverse Transcription Kit (Thermo Scientific). The resulting cDNA was pre-amplified for 12 cycles using pooled miRNA-specific primer-probe sets and the TaqMan PreAmp Mastermix (Thermo Scientific). Quantitative PCR reactions were performed in a LightCycler 480 Real-Time PCR System (Roche Diagnostics, Mannheim, Germany) in a total volume of $10 \mu \mathrm{L}$ containing $1 \mu \mathrm{L}$ of pre-amplified cDNA (1:5 pre-diluted), $0.5 \mu \mathrm{L}$ of miRNA specific primer-probe sets, $5 \mu \mathrm{L}$ of the GoTaq probe qPCR master mix (Promega) and $3.5 \mu \mathrm{L}$ of nuclease-free water. All reactions were measured in duplicate with the following conditions: initial denaturation at $95^{\circ} \mathrm{C}$ for $10 \mathrm{~min}$ followed by 45 cycles at $95^{\circ} \mathrm{C}$ for $15 \mathrm{~s}$ and $60^{\circ} \mathrm{C}$ for $60 \mathrm{~s}$. Threshold cycles $(\mathrm{Ct})$ were determined by the second derivative method and then averaged. For relative quantification, every sample was analyzed for the endogenous reference miRNA miR-16-5p. Baseline and threshold settings were constant across the complete experimental series. Reactions were regarded as valid when the threshold cycle $\mathrm{Ct}$ of miR-16-5p was within the range of 13-23. Expression levels were calculated by applying the $\Delta \mathrm{Ct}$ method [18], given as the $\Delta \mathrm{Ct}$ between reference and test miRNA. By using the normfinder script, we could confirm that in our patient cohort, miR-16-5p gene expression is stable enough to be considered as a reference gene (normfinder stability value 0.08 ). Any reactions that did not show any amplification were manually set to $\mathrm{Ct} 40$ if the $\mathrm{Ct}$ of the reference miRNA miR-16-5p of the respective sample was within the range of 13-23. All reactions were performed blinded to the study end points.

\subsection{Statistical Methods}

Differences in the clinical factors and serum miRNA expression values were analyzed using chi-square (factor variables) or nonparametric Mann-Whitney (continuous variables) statistical tests. The predictive modeling was performed using regularized generalized logistic regression modeling as implemented in the glmnet package for R. A fitted probability of $50 \%$ was used as the cut-off for assigning the binomial group labels. Receiver 
operator characteristics (ROC) were calculated using the pROC package. For the estimation of the benefit of predictive models we used the decision curve analysis method [19]. The net expected regret difference and individual thresholds of 20 clinical urologists were calculated based on the regret theory as described [20]. To determine reference gene stability, we used the normfinder script [21]). All calculations were performed with the $\mathrm{R}$ statistical framework Ver. 3.2.1 (R Foundation for Statistical Computing, Vienna, Austria. http://www.R-project.org/).

\section{Results}

We acquired a total of 289 patients consisting of a discovery cohort $(n=80)$ and a validation cohort $(n=209)$ undergoing targeted MRI-ultrasound fusion-guided prostate biopsy. The patients' characteristics and univariate association of the characteristics with the clinical end points PCa and significant PCa with a Gleason score $\geq 7 \mathrm{a}$ are summarized in Table 1; a detailed summary of tumor diagnosis within PI-RADS lesions is shown in Supplementary Table S1. We have found csPCa both in systematic and in targeted biopsies. There were differences in the distribution of the number of patients undergoing primary or repeat MRI-ultrasound fusion-guided biopsy and the distribution of PI-RADS scores (both $p<0.001$; chi-squared test) between the discovery and the validation cohort, whereas patient age, pre-biopsy PSA level, PCa detection rates, and the distribution of biopsy Gleason scores were not significantly different between the two patient cohorts.

As part of a variable selection process, we tested whether individual miRNAs are able to improve the accuracy of tumor prediction, when introduced into a regularized binary logistic regression model. One miRNA, miR-21-5p, failed to pass our internal quality control during data pre-processing and thus was omitted from further analyses.

In the discovery cohort $(n=80)$, two patients who were tumor-negative in the mpMRIultrasound fusion-guided biopsy already had an earlier diagnosis of PCa and were therefore regarded as tumor-positive in this analysis. Each miRNA was added separately to the baseline model and model characteristics were determined (Table 2; upper part). Four out of the seven miRNAs (miR-210-3p, miR-375-3p, miR-486-5p, and let-7c-5p) provided an advantage over the baseline model, quantified by either a gain in PPV/NPV or an increase in the area under the ROC curve (AUC).

We then applied the established regression models to the validation cohort $(n=209)$, and observed that all four identified miRNAs (miR-210-3p, miR-375-3p miR-486-5p, and let-7c-5p) also resulted in a gain in predictive accuracy in the independent validation cohort (Table 2; lower part).

Next, we tested the classification capabilities of the multivariate predictive model. The model consisting of clinical variables (patient age, pre-biopsy PSA level, previous biopsy procedure, and highest PI-RADS score) and miRNAs (miR-210-3p, miR-375-3p, miR-486-5p and let-7c-5p) achieved a PPV of $70.9 \%$ and an NPV of $68.6 \%$. Detailed information about the model and the predictive performance is provided in Supplementary Table S2. The calibration plot of the regression model is shown in Supplementary Figure S1.

Finally, we explored the possibility to utilize clinical information and miRNA expression values to construct a binary regression model to discriminate between tumor-free or insignificant (Gleason score 6 corresponding to Grade group 1) and csPCa. This information might become especially useful in patients with lesions of PI-RADS $\leq 3$ when clinicians have to decide whether to perform a biopsy or not. Two patients with confirmed PCa had a tumor-free biopsy result and were excluded from this analysis. We focused this approach on the patient subgroup with lesions of PI-RADS 1,2, or 3, resulting in a final cohort size of 89. It is noteworthy that 21 of these patients were diagnosed with a csPCa of Gleason score $\geq 7$ a. Moreover, we noticed that two miRNAs (miR-210-3p and miR-375-3p) were present at rather low levels in patient serum, thereby resulting in a considerable number of "undetected" results. Therefore, in order to define a miRNA panel with a potential applicability in routine laboratory practice, we decided to further reduce the incorporated miRNA markers. The final predictive model was constructed using 3 clinical variables 
(patient age, pre-biopsy PSA level, and previous biopsy procedure) and two miRNAs (miR-486-5p and let-7c-5p).

Table 1. Patients' characteristics and univariate association of individual variables with clinical end points.

\begin{tabular}{|c|c|c|c|c|c|}
\hline Parameter & $\begin{array}{c}\text { Discovery } \\
\text { Cohort }(n=80)\end{array}$ & $\begin{array}{c}\text { Validation } \\
\text { Cohort }(n=209)\end{array}$ & $\begin{array}{l}\text { Complete Cohort } \\
\qquad(n=289)\end{array}$ & $\begin{array}{l}\text { Clinical end Point: PCa } \\
\text { P; Relative Risk ( } 95 \% \text { CI) }\end{array}$ & $\begin{array}{c}\text { Clinical end Point: } \\
\text { Significant PCa } \\
\text { P; Relative Risk (95\%CI) }\end{array}$ \\
\hline $\begin{array}{c}\text { Patient age; median } \\
\text { (IQR) }\end{array}$ & $66(59.5-72.25)$ & $65(60-71)$ & $66(60-72)$ & $\begin{array}{c}<0.01 \\
1.08(1.05-1.21)\end{array}$ & $\begin{array}{c}<0.01 \\
1.08(1.05-1.12)\end{array}$ \\
\hline $\begin{array}{l}\text { Pre-biopsy PSA level } \\
\text { (ng/mL; median, IQR) }\end{array}$ & $8.2(6.8-12.5)$ & $8.2(6.0-13.2)$ & $8.19(6.1-13.1)$ & $\begin{array}{c}<0.01 \\
1.06(1.03-1.07)\end{array}$ & $\begin{array}{c}<0.01 \\
1.08(1.04-1.13)\end{array}$ \\
\hline Previous biopsy; N (\%) & & & & $\begin{array}{c}0.35 \\
1.28(0.76-2.17)\end{array}$ & $\begin{array}{c}0.22 \\
1.39(0.82-2.35)\end{array}$ \\
\hline $\begin{array}{l}\text { No } \\
\text { Yes }\end{array}$ & $\begin{array}{l}34(42.5) \\
46(57.5)\end{array}$ & $\begin{array}{c}44(21.0) \\
165(79.0)\end{array}$ & $\begin{array}{l}78(27.0) \\
211(73.0)\end{array}$ & & \\
\hline $\begin{array}{l}\text { Highest PI-RADS } \\
\text { score; N (\%) }\end{array}$ & & & & $\begin{array}{c}<0.01 \\
2.07(1.55-2.79)\end{array}$ & $\begin{array}{c}<0.01 \\
2.39(1.76-3.33)\end{array}$ \\
\hline $\begin{array}{l}1 \\
2 \\
3 \\
4 \\
5\end{array}$ & $\begin{array}{c}1(1.3) \\
0(0) \\
8(10.0) \\
49(61.2) \\
22(27.5)\end{array}$ & $\begin{array}{c}0(0) \\
24(11.5) \\
57(27.3) \\
84(40.2) \\
44(21.0)\end{array}$ & $\begin{array}{c}1(0.4) \\
24(8.3) \\
65(22.5) \\
133(46.0) \\
66(22.8)\end{array}$ & & \\
\hline $\begin{array}{l}\text { Biopsy Gleason score } \\
\text { (GS); N (\%) }\end{array}$ & & & & n.c. & n.c. \\
\hline $\begin{array}{c}\text { Tumor free } \\
6 \\
\text { 7a }(\mathrm{GS} 3+4) \\
\text { 7b (GS4+3) } \\
8 \\
9 \\
10\end{array}$ & $\begin{array}{l}38(47.5) \\
9(11.2) \\
16(20.0) \\
9(11.2) \\
2(2.5) \\
5(6.3) \\
1(1.3)\end{array}$ & $\begin{array}{c}103(49.3) \\
17(8.1) \\
49(23.4) \\
9(4.3) \\
11(5.3) \\
20(9.6) \\
0(0)\end{array}$ & $\begin{array}{l}141(48.8) \\
26(9.0) \\
65(22.5) \\
18(6.2) \\
13(4.5) \\
25(8.7) \\
1(0.3)\end{array}$ & & \\
\hline $\begin{array}{c}\text { Targeted biopsy cores; } \\
\text { median (IQR) }\end{array}$ & $3(2-4)$ & $6(4-7)$ & $5(3-6)$ & $\begin{array}{c}0.12 \\
0.93(0.85-1.03)\end{array}$ & $\begin{array}{c}0.98 \\
0.98(0.89-1.08)\end{array}$ \\
\hline $\begin{array}{c}\text { Systematic biopsy } \\
\text { cores; median (IQR) }\end{array}$ & $9(8-11)$ & $12(12-12)$ & $12(11-12)$ & $0.99(0.91-1.08)$ & $0.99(0.91-1.08)$ \\
\hline Tumor status; N (\%) & & & & n.c. & n.c. \\
\hline $\begin{array}{l}\text { No tumor } \\
\text { Tumor }\end{array}$ & $\begin{array}{c}36(45.0) \\
44^{1}(55.0)\end{array}$ & $\begin{array}{l}103(49.3) \\
106(50.7)\end{array}$ & $\begin{array}{c}139(48.1) \\
150^{1}(51.9)\end{array}$ & & \\
\hline $\begin{array}{l}\Delta \mathrm{Ct} \text { miR-141-3p; } \\
\text { median (IQR) }\end{array}$ & $18.4(15.2-18.4)$ & $16.7(15.0-19.6)$ & $16.9(15.1-20.3)$ & $\begin{array}{c}0.46 \\
1.03(0.96-1.10)\end{array}$ & $\begin{array}{c}0.97 \\
1.00(0.93-1.08)\end{array}$ \\
\hline $\begin{array}{l}\Delta \mathrm{Ct} \text { miR-375-5p; } \\
\text { median (IQR) }\end{array}$ & 16.5 (14.4-19.1) & $14.7(13.4-19.2)$ & $15.3(13.6-19.2)$ & $\begin{array}{c}0.31 \\
1.04(0.97-1.11)\end{array}$ & $0.99(0.93-1.06)$ \\
\hline $\begin{array}{l}\Delta \mathrm{Ct} \text { miR-21-5p; } \\
\text { median (IQR) }\end{array}$ & $9.2(8.8-9.8)$ & $7.4(7.0-8.0)$ & $7.9(7.2-8.8)$ & $\begin{array}{c}0.74 \\
1.03(0.87-1.21)\end{array}$ & $\begin{array}{c}0.61 \\
0.96(0.80-1.13)\end{array}$ \\
\hline $\begin{array}{c}\Delta \mathrm{Ct} \text { miR-320b; median } \\
\text { (IQR) }\end{array}$ & $9.8(8.6-10.7)$ & $10.2(9.8-10.7)$ & $10.2(9.8-10.7)$ & $0.89(0.74-1.05)$ & $\begin{array}{c}0.18 \\
0.88(0.74-1.05)\end{array}$ \\
\hline $\begin{array}{c}\Delta \mathrm{Ct} \text { miR-210-3p; } \\
\text { median (IQR) }\end{array}$ & $12.8(12.3-14.8)$ & 12.7 (11.9-13.7) & $12.7(12.1-13.8)$ & $\begin{array}{c}0.36 \\
0.97(0.89-1.04)\end{array}$ & $0.95(0.88-1.03)$ \\
\hline $\begin{array}{c}\Delta C \text { t let-7c-5p; median } \\
\text { (IQR) }\end{array}$ & $11.1(10.7-11.5)$ & $11.7(11.1-12.5)$ & $11.5(11.0-12.2)$ & $1.12(0.97-1.32)$ & $\begin{array}{c}0.39 \\
1.07(0.92-1.23)\end{array}$ \\
\hline $\begin{array}{l}\Delta \mathrm{Ct} \text { miR-486-5p; } \\
\text { median (IQR) }\end{array}$ & $3.2(2.8-3.5)$ & $3.1(2.8-3.4)$ & $3.1(2.8-3.5)$ & $\begin{array}{c}0.482 \\
1.15(0.78-1.79)\end{array}$ & $\begin{array}{c}0.70 \\
0.92(0.60-1.37)\end{array}$ \\
\hline
\end{tabular}

IQR-interquartile range; ${ }^{1}$ Two patients with a tumour-free biopsy had histologically confirmed prostate cancer; n.c.- not calculated.

The highest Youden index was determined at a cut-off of $14 \%$. To determine if this cut-off value of $14 \%$ represents a clinically reasonable threshold value, we interviewed 20 clinical decision makers and calculated individual threshold cut-off values [20] based on personal experience. The median of individual threshold values was $12 \%$ (interquartile range $8-28 \%$ ). This demonstrated that our predictive model is able to provide additional information within a range of threshold values that are applicable in clinical practice.

By classifying at a cut-off value of $14 \%$, a total of 20 of the 21 csPCa cases were correctly identified. This high sensitivity of $95.2 \%$ of csPCa cases identified was accompanied by an NPV of $97.1 \%$ for the absence of csPCa. Detailed information about the predictive mathematical model and the predictive performance is provided in Table 3 . The calibration plot of the regression model is shown in Supplementary Figure S2 and a receiver-operator curve comparison with the baseline model is given in Supplementary Figure S3. A decision curve analysis was used to quantify the benefit of applying the predictive model. At the specified threshold of $14 \%$ risk, the net benefit of the model accounts to $16.1 \%$ (Figure 1 ). Following a related method, the regret theory, we sought to more exactly determine the 
range of threshold values where our predictive model might provide a benefit for patients. Following the published equations [20], we calculated the Net Expected Regret Difference. Up to a threshold cut-off of $6 \%$, our model does not provide a benefit over the strategy of biopsy all patients and above a cut-off of $98 \%$, it does not provide a benefit over the strategy of biopsy no patient. Between $7 \%$ and $97 \%$, our model provided a benefit over both extreme strategies (Figure 2 and Supplementary Table S3).

Table 2. Predictive performance of clinical parameters and miRNAs in the discovery and validation cohorts.

\begin{tabular}{|c|c|c|c|c|c|c|}
\hline \multicolumn{7}{|c|}{ Model Establishment: Discovery Cohort } \\
\hline \multicolumn{7}{|c|}{ Parameters of the Model } \\
\hline Clinical parameters & miRNA & PPV (\%) & NPV (\%) & Sensitivity (\%) & Specificity (\%) & AUC \\
\hline Age, PSA, Prev. biopsy, PI-RADS & & 66.0 & 66.7 & 79.5 & 50.0 & 0.684 \\
\hline Age, PSA, Prev. biopsy, PI-RADS & $\operatorname{miR}-141-3 p$ & 64.8 & 65.3 & 79.5 & 52.7 & 0.684 \\
\hline Age, PSA, Prev. biopsy, PI-RADS & $\operatorname{miR}-375-3 p$ & 64.8 & 65.3 & 79.5 & 52.8 & 0.689 \\
\hline Age, PSA, Prev. biopsy, PI-RADS & miR-21-5p & 68.0 & 66.7 & 77.3 & 55.5 & 0.718 \\
\hline Age, PSA, Prev. biopsy, PI-RADS & miR-320b & 64.1 & 62.9 & 77.3 & 52.8 & 0.681 \\
\hline Age, PSA, Prev. biopsy, PI-RADS & miR-210-3p & 67.3 & 67.8 & 79.5 & 52.8 & 0.689 \\
\hline Age, PSA, Prev. biopsy, PI-RADS & let-7c-5p & 86.0 & 68.7 & 77.3 & 61.1 & 0.707 \\
\hline Age, PSA, Prev. biopsy, PI-RADS & $\operatorname{miR}-486-5 p$ & 70.8 & 66.7 & 77.3 & 55.5 & 0.697 \\
\hline \multicolumn{7}{|c|}{$\begin{array}{c}\text { Model Validation: Validation Cohort } \\
\text { Parameters of the Model }\end{array}$} \\
\hline Clinical parameters & miRNA & PPV (\%) & NPV (\%) & Sensitivity (\%) & Specificity (\%) & AUC \\
\hline Age, PSA, Prev. biopsy, PI-RADS & & 65.4 & 65.6 & 67.9 & 63.1 & 0.732 \\
\hline Age, PSA, Prev. biopsy, PI-RADS & miR-141-3p & 66.7 & 60.3 & 67.9 & 65.0 & 0.734 \\
\hline Age, PSA, Prev. biopsy, PI-RADS & $\operatorname{miR}-375-3 p$ & 68.1 & 68.7 & 70.8 & 66.0 & 0.736 \\
\hline Age, PSA, Prev. biopsy, PI-RADS & $\operatorname{miR}-21-5 p$ & 75.6 & 54.6 & 26.4 & 91.3 & 0.702 \\
\hline Age, PSA, Prev. biopsy, PI-RADS & $\operatorname{miR}-320 b$ & 69.3 & 66.7 & 66.0 & 66.7 & 0.737 \\
\hline Age, PSA, Prev. biopsy, PI-RADS & miR-210-3p & 65.1 & 65.9 & 68.9 & 62.1 & 0.733 \\
\hline Age, PSA, Prev. biopsy, PI-RADS & let-7c-5p & 63.8 & 66.7 & 71.7 & 58.3 & 0.734 \\
\hline Age, PSA, Prev. biopsy, PI-RADS & $\operatorname{miR}-486-5 p$ & 65.7 & 58.7 & 65.1 & 65.0 & 0.719 \\
\hline
\end{tabular}

Table 3. Comparison of pathological and predicted outcome; covariates and regression coefficients of the regression model for predicting significant PCa in patients with PI-RADS scores 1, 2, and 3. For binomial classification, the optimized threshold of $14 \%$ risk was used.

\begin{tabular}{|c|c|c|c|c|}
\hline \multirow{5}{*}{ Pathological disease status } & \multicolumn{4}{|c|}{ Predicted Disease Status } \\
\hline & & $\begin{array}{c}\text { Tumor- } \\
\text { free/Gleason } \\
6\end{array}$ & Gleason $7 a-10$ & \\
\hline & Tumor-free/Gleason $6^{*}$ & 33 & 35 & Specificity $48.5 \%$ \\
\hline & Gleason 7a-10 * & 1 & 20 & Sensitivity $95.2 \%$ \\
\hline & & NPV 97.1\% & PPV $36.4 \%$ & \\
\hline Covariate & Model coefficient & & & \\
\hline Intercept & -6.47144586 & & & \\
\hline Patient age & 0.06001647 & & & \\
\hline Pre-biopsy PSA level & 0.09607283 & & & \\
\hline Previous biopsy procedure & -1.36328590 & & & \\
\hline miR-486-5p & -0.52307176 & & & \\
\hline let-7c-5p & 0.30509631 & & & \\
\hline
\end{tabular}

* Tumor-free/Gleason 6 corresponding to Tumor-free/Grade group 1; Gleason 7a-10 corresponding to Grade groups $\geq 2$. 


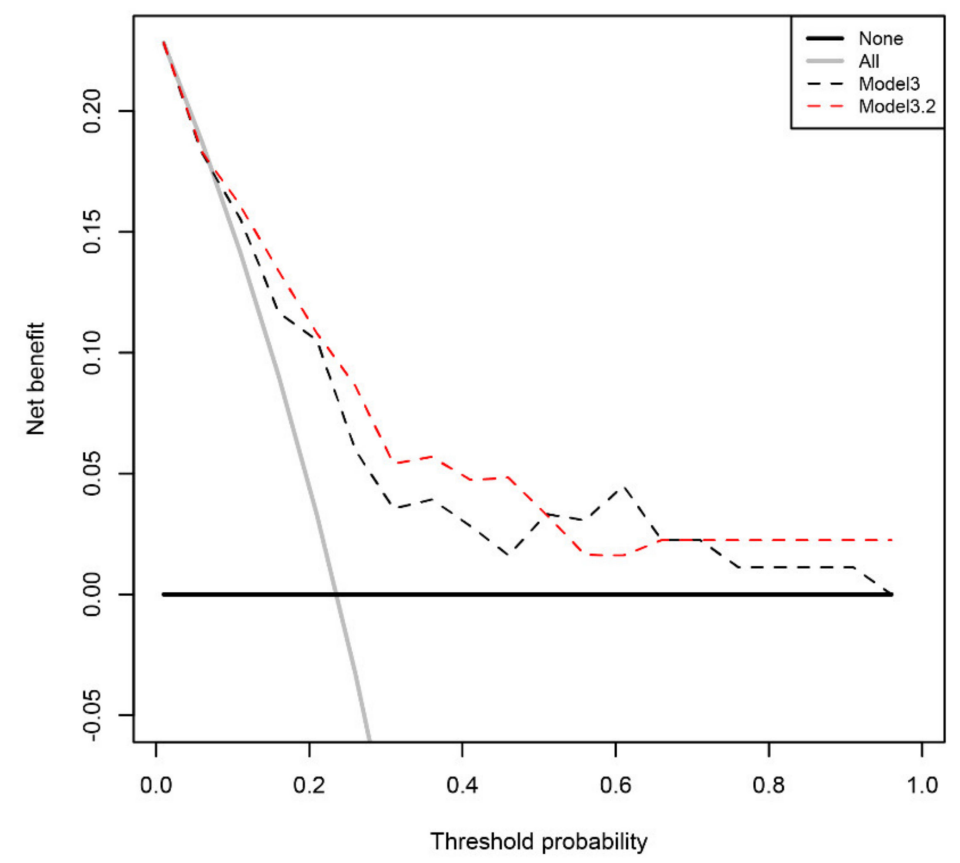

Figure 1. Decision curve analysis. The net benefit of using the mathematical models for biopsy indication instead of the extreme strategies is shown. Model3 includes PSA level, patient age and previous biopsy status, model3.2 includes PSA level, patient age, previous biopsy status, and the expression levels of miR-486 and let-7c. Model3.2 provides a benefit over the extreme strategies of recommending a biopsy in all patients or recommending a biopsy for no patient within a threshold range of $7 \%$ to $97 \%$.

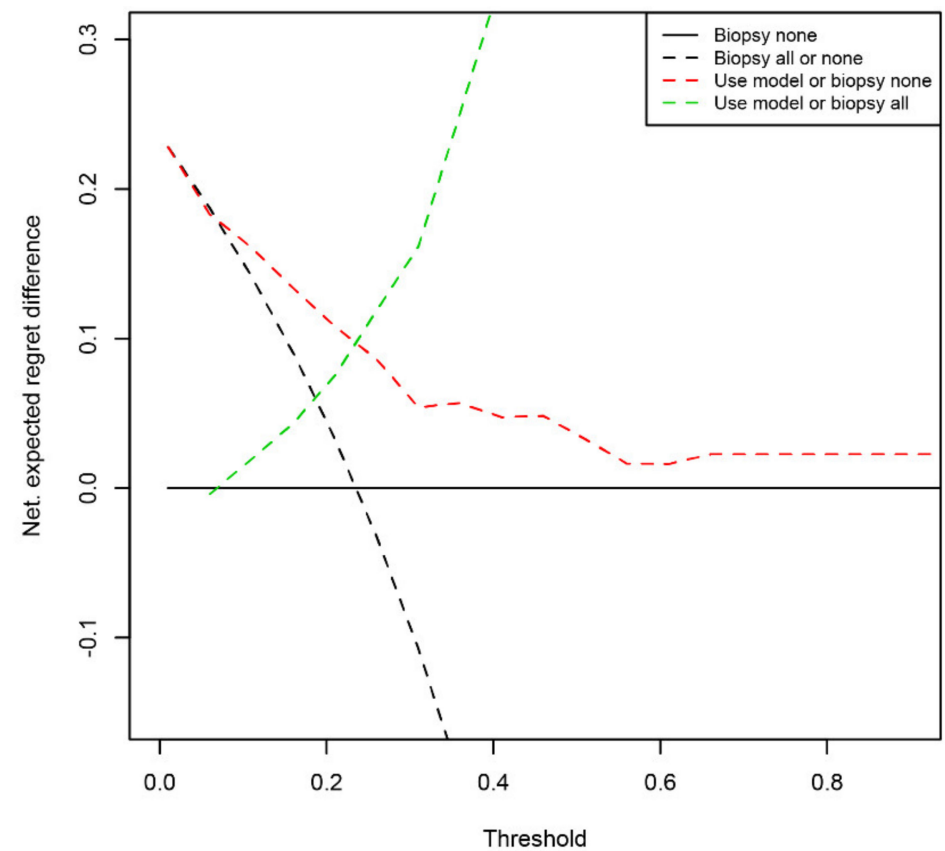

Figure 2. Expected Regret Difference. The net Expected Regret Difference of using the model3.2 for biopsy indication in pair-wise comparison. Within a threshold range of $7 \%$ and $97 \%$, our model provided a benefit over both extreme strategies.

\section{Discussion}

A recent nationwide survey among clinicians in Germany underscores the widespread acceptance of mpMRI and targeted prostatic biopsies [5]. Despite the gain in diagnostic 
accuracy by mpMRI, there is still an on-going effort to utilize this information for risk classification. However, the combination of clinical parameters and mpMRI is a promising tool to reduce unnecessary prostate biopsies [22]. Here, we tested the additional predictive value of miRNAs incorporated in a regression model to predict PCa detection and tumor aggressiveness prior to mpMRI-ultrasound fusion-guided biopsy. MiRNAs were selected because of their differential expression in PCa tissue compared to non-malignant prostatic tissue $[8,9]$. Our study shows that it is possible to enhance the prediction of $\mathrm{PCa}$ detection by adding serum miRNA expression levels to clinical data and the PI-RADS score. However, up to now, there is a strong suggestion that PI-RADS 3 lesions should also be biopsied [5,23], because they might harbor significant PCa ranging from $12 \%$ [24] to $34 \%$ in current cohorts [25], even though other studies show only a limited incidence of significant PCa [26]. Yang et al. utilized mpMRI imaging information and routine clinical parameters for modeling cancer risk particularly in patients with PI-RADS 3 lesions and suggested their model for the reduction of unnecessary biopsies [27]. Interestingly, our mathematical models performed very well at identifying patients with csPCa of Gleason score $\geq 7$ a especially in the subgroup of patients with lesions of PI-RADS $\leq 3$. We could identify patients with csPCa with a sensitivity of $95.2 \%$ while at the same time predicting the absence of csPCa with an NPV of $97.1 \%$ These properties of the model might be of high interest for clinicians to identify those patients that can be spared a prostate biopsy (high NPV) while still being able to detect the majority of significant PCa cases (high sensitivity).

Interestingly, none of the incorporated serum-miRNAs was significantly associated with PCa diagnosis or Gleason score by itself, although all the measured miRNAs have been described to be differentially expressed in PCa tissue and are suggested to play a role in prostatic carcinogenesis $[8,9,12,28-31]$. Moreover, we showed in previous studies that clinical parameters, such as the pre-biopsy PSA level, Gleason score, or TNM status, have only a limited impact on the overall abundance of miRNAs in the patients' blood [10].

Molecular tests, such as the PCA3 test and others, have the potential to enhance such predictive models. Fenstermaker et al. demonstrated that a positive PCA3 test was associated with PCa detection in MRI-ultrasound fusion-guided targeted biopsies in patients with visible lesions in the primary setting of PCa detection [32]. However, the impact in highly suspicious lesions was limited in their study. They propose to test the value of the PCA3 test particularly in patients with low or equivocal PCa probability after mpMRI. With the proposed cut-off of 35, the PCA3 test exhibited a sensitivity of $62 \%$ and $73 \%$ as well as a NPV of $88 \%$ and $91 \%$ in patients with lesions of PI-RADS 2 and 3, respectively [32]. In the setting of primary, not mpMRI fusion-supported prostate biopsies, several studies evaluated molecular tests such as the PCA3 test or the prostate health index (PHI) and their abilities to predict the diagnostic outcome. Thereby, the PHI performed better than PSA alone with sensitivities ranging from $74-94 \%$ and specificities ranging from $49-72 \%[33,34]$. In future studies, prostate volume, PSA density, number, and the exact localization of the MRI foci might be considered as well. In addition, in a future prospective clinical trial a centralized pathology review and a centralized radiological review are necessary.

One of the most recent molecular diagnostic tests described is the STHLM3 model [35]. This model combines several plasma protein biomarkers, genetic polymorphisms, and clinical information. The STHLM3 model could identify a population where the tumor detection rate reached more than 50\% [35]. Nevertheless, in our highly selected patient cohort, our mathematical model correctly predicted a PCa diagnosis in $72 \%$ of cases.

\section{Conclusions}

In summary, our findings demonstrate that the addition of miRNA expression information to MRI imaging and clinical data is able to enhance the tumor prediction rate. Particularly for patients with PI-RADS $\leq 3$ lesions, the miRNA-supported predictive models perform very well at predicting a clinically significant PCa. 
Supplementary Materials: The following are available online at https://www.mdpi.com/article/10 .3390 / cells10061315/s1, Figure S1: Calibration plot of the optimized regression model for predicting tumor status; Figure S2: Calibration plot of the optimized regression model for predicting significant PCa in patients with PI-RADS scores 1, 2, and 3; Figure S3: Receiver operator curve comparison. Table S1: Number of patients with corresponding Gleason scores and PI-RADS scores; Table S2: Comparison of pathological and predicted outcome; Covariates and regression coefficients of the optimized regression model for predicting tumor status; Table S3: Net expected regret differences at defined threshold cut-off values. Pair-wise comparisons of regret differences for the detection of significant PCa in patients with lesions of PI-RADS $\leq 3$.

Author Contributions: Conceptualization, B.K., H.T., S.F., and S.W.; investigation, K.E., J.P., and T.J.; data curation, A.B., J.P., T.J., M.F., A.K., M.W., J.H., F.K., D.S., A.C., M.H., I.P., A.H., and G.B.; formal analysis, H.T., K.E., S.F., and S.W.; writing-original draft preparation, B.K., H.T., B.W., and S.W.; writing-review and editing, K.E., S.F., and A.B. All authors have read and agreed to the published version of the manuscript.

Funding: S.W. was supported by the Wilhelm Sander-Stiftung (Grant ID 2015.171.1) and the Johannes und Frieda Marohn-Stiftung (Grant ID Alz/Iko-Härt/2016). H.T. was supported by the Deutsche Forschungsgemeinschaft (Grant ID TA145/17-1). The funding sources had no involvement in study design; in collection, analysis, and interpretation of data; or the writing of the report.

Institutional Review Board Statement: Ethical approval was provided by the ethics institutional review board of the University Hospital Erlangen (No. 3755, dated February 2008) and by the ethics institutional review board of the Technische Universität Dresden (No. EK194092004, dated July 2009).

Informed Consent Statement: Written informed consent was obtained before biopsy and the study was performed according to the Declaration of Helsinki.

Data Availability Statement: Data is contained within this article and the Supplementary Materials. Further data can be obtained from the corresponding author upon reasonable request.

Acknowledgments: We thank American Journal Experts for providing language editing service. We thank Katrin Weigelt, Lena Scheinost, and Andrea Lohse-Fischer for excellent technical assistance.

Conflicts of Interest: The authors declare no competing interests.

\section{References}

1. Mottet, N.; Bellmunt, J.; Bolla, M.; Briers, E.; Cumberbatch, M.G.; De Santis, M.; Fossati, N.; Gross, T.; Henry, A.M.; Joniau, S.; et al. EAU-ESTRO-SIOG Guidelines on Prostate Cancer. Part 1: Screening, Diagnosis, and Local Treatment with Curative Intent. Eur. Urol. 2017, 71, 618-629. [CrossRef] [PubMed]

2. Sinnott, M.; Falzarano, S.M.; Hernandez, A.V.; Jones, J.S.; Klein, E.A.; Zhou, M.; Magi-Galluzzi, C. Discrepancy in prostate cancer localization between biopsy and prostatectomy specimens in patients with unilateral positive biopsy: Implications for focal therapy. Prostate 2012, 72, 1179-1186. [CrossRef]

3. Jones, J.S.; Patel, A.; Schoenfield, L.; Rabets, J.C.; Zippe, C.D.; Magi-Galluzzi, C. Saturation technique does not improve cancer detection as an initial prostate biopsy strategy. J. Urol. 2006, 175, 485-488. [CrossRef]

4. Siddiqui, M.M.; Rais-Bahrami, S.; Turkbey, B.; George, A.K.; Rothwax, J.; Shakir, N.; Okoro, C.; Raskolnikov, D.; Parnes, H.L.; Linehan, W.M.; et al. Comparison of MR/ultrasound fusion-guided biopsy with ultrasound-guided biopsy for the diagnosis of prostate cancer. JAMA 2015, 313, 390-397. [CrossRef]

5. Saar, M.; Linxweiler, J.; Borkowetz, A.; Fussek, S.; Urbanova, K.; Bellut, L.; Kristiansen, G.; Wullich, B.; German Prostate Cancer, C. Current Role of Multiparametric MRI and MRI Targeted Biopsies for Prostate Cancer Diagnosis in Germany: A Nationwide Survey. Urol. Int. 2020, 104, 731-740. [CrossRef]

6. Cash, H.; Maxeiner, A.; Stephan, C.; Fischer, T.; Durmus, T.; Holzmann, J.; Asbach, P.; Haas, M.; Hinz, S.; Neymeyer, J.; et al. The detection of significant prostate cancer is correlated with the Prostate Imaging Reporting and Data System (PI-RADS) in MRI/transrectal ultrasound fusion biopsy. World J. Urol. 2016, 34, 525-532. [CrossRef] [PubMed]

7. Junker, D.; Quentin, M.; Nagele, U.; Edlinger, M.; Richenberg, J.; Schaefer, G.; Ladurner, M.; Jaschke, W.; Horninger, W.; Aigner, F. Evaluation of the PI-RADS scoring system for mpMRI of the prostate: A whole-mount step-section analysis. World J. Urol. 2015, 33, 1023-1030. [CrossRef]

8. Szczyrba, J.; Loprich, E.; Wach, S.; Jung, V.; Unteregger, G.; Barth, S.; Grobholz, R.; Wieland, W.; Stohr, R.; Hartmann, A.; et al. The microRNA profile of prostate carcinoma obtained by deep sequencing. Mol. Cancer Res. 2010, 8, 529-538. [CrossRef] [PubMed]

9. Wach, S.; Nolte, E.; Szczyrba, J.; Stohr, R.; Hartmann, A.; Orntoft, T.; Dyrskjot, L.; Eltze, E.; Wieland, W.; Keck, B.; et al. MicroRNA profiles of prostate carcinoma detected by multiplatform microRNA screening. Int. J. Cancer 2012, 130, 611-621. [CrossRef] [PubMed] 
10. Leidinger, P.; Hart, M.; Backes, C.; Rheinheimer, S.; Keck, B.; Wullich, B.; Keller, A.; Meese, E. Differential blood-based diagnosis between benign prostatic hyperplasia and prostate cancer: miRNA as source for biomarkers independent of PSA level, Gleason score, or TNM status. Tumour Biol. 2016, 37, 10177-10185. [CrossRef]

11. Wach, S.; Al-Janabi, O.; Weigelt, K.; Fischer, K.; Greither, T.; Marcou, M.; Theil, G.; Nolte, E.; Holzhausen, H.J.; Stohr, R.; et al. The combined serum levels of miR-375 and urokinase plasminogen activator receptor are suggested as diagnostic and prognostic biomarkers in prostate cancer. Int. J. Cancer 2015, 137, 1406-1416. [CrossRef] [PubMed]

12. Haldrup, C.; Kosaka, N.; Ochiya, T.; Borre, M.; Hoyer, S.; Orntoft, T.F.; Sorensen, K.D. Profiling of circulating microRNAs for prostate cancer biomarker discovery. Drug Deliv. Transl. Res. 2014, 4, 19-30. [CrossRef]

13. Song, C.; Chen, H.; Wang, T.; Zhang, W.; Ru, G.; Lang, J. Expression profile analysis of microRNAs in prostate cancer by next-generation sequencing. Prostate 2015, 75, 500-516. [CrossRef]

14. Lieb, V.; Weigelt, K.; Scheinost, L.; Fischer, K.; Greither, T.; Marcou, M.; Theil, G.; Klocker, H.; Holzhausen, H.J.; Lai, X.; et al. Serum levels of miR-320 family members are associated with clinical parameters and diagnosis in prostate cancer patients. Oncotarget 2017, 9, 10402-10416. [CrossRef]

15. Mahn, R.; Heukamp, L.C.; Rogenhofer, S.; von Ruecker, A.; Müller, S.C.; Ellinger, J. Circulating microRNAs (miRNA) in serum of patients with prostate cancer. Urology 2011, 77, 1265.e9-1265.e16. [CrossRef]

16. Borkowetz, A.; Platzek, I.; Toma, M.; Laniado, M.; Baretton, G.; Froehner, M.; Koch, R.; Wirth, M.; Zastrow, S. Comparison of systematic transrectal biopsy to transperineal magnetic resonance imaging/ultrasound-fusion biopsy for the diagnosis of prostate cancer. BJU Int. 2015, 116, 873-879. [CrossRef]

17. Mottet, N.; Bellmunt, J.; Briers, E.; Bolla, M.; Bourke, L.; Cornford, P.; De Santis, M.; Henry, A.; Joniau, S.; Lam, T.; et al. EAU-ESTRO-ESUR-SIOG Guidelines on Prostate Cancer. In Proceedings of the at the EAU Annual Congress, Milan, Italy, 9-12 July 2021; EAU Guidelines Office: Arnhem, The Netherlands, 2021; ISBN 978-94-92671-13-4.

18. Schmittgen, T.D.; Livak, K.J. Analyzing real-time PCR data by the comparative C(T) method. Nat. Protoc. 2008, 3, 1101-1108. [CrossRef] [PubMed]

19. Vickers, A.J.; Elkin, E.B. Decision curve analysis: A novel method for evaluating prediction models. Med. Decis. Making 2006, 26, 565-574. [CrossRef] [PubMed]

20. Tsalatsanis, A.; Hozo, I.; Vickers, A.; Djulbegovic, B. A regret theory approach to decision curve analysis: A novel method for eliciting decision makers' preferences and decision-making. BMC Med. Inform. Decis. Mak. 2010, 10, 51. [CrossRef]

21. Andersen, C.L.; Jensen, J.L.; Ørntoft, T.F. Normalization of real-time quantitative reverse transcription-PCR data: A model-based variance estimation approach to identify genes suited for normalization, applied to bladder and colon cancer data sets. Cancer Res. 2004, 64, 5245-5250. [CrossRef]

22. Radtke, J.P.; Wiesenfarth, M.; Kesch, C.; Freitag, M.T.; Alt, C.D.; Celik, K.; Distler, F.; Roth, W.; Wieczorek, K.; Stock, C.; et al. Combined Clinical Parameters and Multiparametric Magnetic Resonance Imaging for Advanced Risk Modeling of Prostate Cancer-Patient-tailored Risk Stratification Can Reduce Unnecessary Biopsies. Eur. Urol. 2017, 72, 888-896. [CrossRef] [PubMed]

23. Luzzago, S.; Musi, G.; Catellani, M.; Russo, A.; Di Trapani, E.; Mistretta, F.A.; Bianchi, R.; Cozzi, G.; Conti, A.; Pricolo, P.; et al. Multiparametric Magnetic-Resonance to Confirm Eligibility to an Active Surveillance Program for Low-Risk Prostate Cancer: Intermediate Time Results of a Third Referral High Volume Centre Active Surveillance Protocol. Urol. Int. 2018, 101, 56-64. [CrossRef] [PubMed]

24. Borkowetz, A.; Platzek, I.; Toma, M.; Renner, T.; Herout, R.; Baunacke, M.; Laniado, M.; Baretton, G.B.; Froehner, M.; Zastrow, S.; et al. Evaluation of Prostate Imaging Reporting and Data System Classification in the Prediction of Tumor Aggressiveness in Targeted Magnetic Resonance Imaging/Ultrasound-Fusion Biopsy. Urol. Int. 2017, 99, 177-185. [CrossRef]

25. Venderink, W.; van Luijtelaar, A.; Bomers, J.G.; van der Leest, M.; Hulsbergen-van de Kaa, C.; Barentsz, J.O.; Sedelaar, J.P.; Futterer, J.J. Results of Targeted Biopsy in Men with Magnetic Resonance Imaging Lesions Classified Equivocal, Likely or Highly Likely to Be Clinically Significant Prostate Cancer. Eur. Urol. 2017, 73, 353-360. [CrossRef]

26. Liddell, H.; Jyoti, R.; Haxhimolla, H.Z. mp-MRI Prostate Characterised PIRADS 3 Lesions are Associated with a Low Risk of Clinically Significant Prostate Cancer-A Retrospective Review of 92 Biopsied PIRADS 3 Lesions. Curr. Urol. 2015, 8, 96-100. [CrossRef]

27. Yang, S.; Zhao, W.; Tan, S.; Zhang, Y.; Wei, C.; Chen, T.; Shen, J. Combining clinical and MRI data to manage PI-RADS 3 lesions and reduce excessive biopsy. Transl. Androl. Urol. 2020, 9, 1252-1261. [CrossRef]

28. Sato, S.; Katsushima, K.; Shinjo, K.; Hatanaka, A.; Ohka, F.; Suzuki, S.; Naiki-Ito, A.; Soga, N.; Takahashi, S.; Kondo, Y. Histone Deacetylase Inhibition in Prostate Cancer Triggers miR-320-Mediated Suppression of the Androgen Receptor. Cancer Res. 2016, 76, 4192-4204. [CrossRef]

29. Yang, Y.; Ji, C.; Guo, S.; Su, X.; Zhao, X.; Zhang, S.; Liu, G.; Qiu, X.; Zhang, Q.; Guo, H.; et al. The miR-486-5p plays a causative role in prostate cancer through negative regulation of multiple tumor suppressor pathways. Oncotarget 2017, 8, 72835-72846. [CrossRef] [PubMed]

30. Kachakova, D.; Mitkova, A.; Popov, E.; Popov, I.; Vlahova, A.; Dikov, T.; Christova, S.; Mitev, V.; Slavov, C.; Kaneva, R. Combinations of serum prostate-specific antigen and plasma expression levels of let-7c, miR-30c, miR-141, and miR-375 as potential better diagnostic biomarkers for prostate cancer. DNA Cell Biol. 2015, 34, 189-200. [CrossRef] 
31. Schubert, M.; Spahn, M.; Kneitz, S.; Scholz, C.J.; Joniau, S.; Stroebel, P.; Riedmiller, H.; Kneitz, B. Distinct microRNA expression profile in prostate cancer patients with early clinical failure and the impact of let-7 as prognostic marker in high-risk prostate cancer. PLoS ONE 2013, 8, e65064. [CrossRef] [PubMed]

32. Fenstermaker, M.; Mendhiratta, N.; Bjurlin, M.A.; Meng, X.; Rosenkrantz, A.B.; Huang, R.; Deng, F.M.; Zhou, M.; Huang, W.C.; Lepor, H.; et al. Risk Stratification by Urinary Prostate Cancer Gene 3 Testing Before Magnetic Resonance Imaging-Ultrasound Fusion-targeted Prostate Biopsy Among Men With No History of Biopsy. Urology 2017, 99, 174-179. [CrossRef] [PubMed]

33. Fuchsova, R.; Topolcan, O.; Windrichova, J.; Hora, M.; Dolejsova, O.; Pecen, L.; Kasik, P.; Novak, J.; Casova, M.; Smejkal, J. PHI in the Early Detection of Prostate Cancer. Anticancer Res. 2015, 35, 4855-4857. [PubMed]

34. Ferro, M.; Bruzzese, D.; Perdona, S.; Mazzarella, C.; Marino, A.; Sorrentino, A.; Di Carlo, A.; Autorino, R.; Di Lorenzo, G.; Buonerba, C.; et al. Predicting prostate biopsy outcome: Prostate health index (phi) and prostate cancer antigen 3 (PCA3) are useful biomarkers. Clin. Chim. Acta 2012, 413, 1274-1278. [CrossRef]

35. Gronberg, H.; Adolfsson, J.; Aly, M.; Nordstrom, T.; Wiklund, P.; Brandberg, Y.; Thompson, J.; Wiklund, F.; Lindberg, J.; Clements, M.; et al. Prostate cancer screening in men aged 50-69 years (STHLM3): A prospective population-based diagnostic study. Lancet Oncol. 2015, 16, 1667-1676. [CrossRef] 